\title{
Flow of Planets Raises Short Period Fall-Off
}

\author{
Stuart F. Taylor ${ }^{1,2}$ \\ ${ }^{1}$ Job Seeking \\ ${ }^{2}$ Participation Worldscope/Global Telescope Science \\ Los Angeles, California, U.S., and Hong Kong, SAR China \\ email: astrostuart@gmail.com
}

\begin{abstract}
After finding more planets than expected at the shortest period, there has been an effort to explain their numbers by weak tidal friction. However, we find that the strength of tidal dissipation that would produce the occurence distribution found from Kepler planet candidates is different for giant versus medium radii planets. This discrepancy can be resolved if there is a "flow" of the largest planets regularly arriving such that they go through a "hot Jupiter" stage. We also show a correlation of higher stellar $\mathrm{Fe} / \mathrm{H}$ with higher eccentricity of giant planets that may be from smaller planets having been sent into the star by the migration of the larger planet. This disruption of the orbits of medium and smaller planets could account for the lower occurrence of "hot Neptune" medium radius planets.
\end{abstract}

Keywords. planetary systems: dynamical evolution, tidal interactions, stars: fundamental parameters

\section{Introduction}

The most unexpected planet finding could be that the number of planets with shorter periods is larger than expected. Most such close planets would been expected to quickly migrate into the star in timescales short relative to the lifetime of the stars unless tidal dissipation is unexpectedly weak, leading to work seeking to explain weak dissipation in stars. We show how the occurrence distribution of differently-sized planets is more consistent with the explanation that these planets have more recently arrived as a flow of inwardly migrating planets, with giant planets more likely to be found while gradually going through stage having the shortest periods. This "flow" of inwardly migrating planets would be the final stage of the high eccentricity migration flow proposed by Socrates et al. (2012; H12 hereafter) to explain the short period pileup of giant planets.

We have previously shown that the shortest period region of the exoplanet occurrence distribution has a fall-off shaped by inward tidal migration due to stellar tides, that is, tides on the star caused by the planets (Taylor 2012; T12 hereafter). The power index of the fall-off of giant ( 8 to 32 earth radii " $R_{\text {Earth" }}$ ) and medium (4 to $8 R_{\text {Earth }}$ ) radii planet candidates found from Kepler data by $\mathrm{H} 12$ is close to $13 / 3$, which is the power index resulting from tidal migration (T12). However, there is a discrepancy of the strength of the tidal migration determined using giant and medium planets that is best resolved by the explanation that more giant than medium radii planets are observed passing through these shortest period orbits.

We also present a correlation between higher eccentricity of planetary orbits with higher $\mathrm{Fe} / \mathrm{H}$ of host stars, which could be explained by high eccentricity planets being associated with recent episodes of other planets into stars. The shortest period planet hosting stars may have already convected the pollution away from the surface. The clearing of other planets by migrating hot giant planets may result in the absence of additional planets in hot Jupiter systems. 


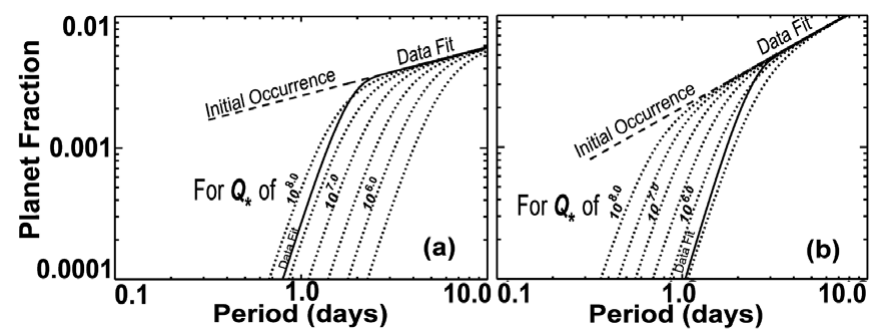

Figure 1. Migrated evolution compared to data for masses and radii for giant (100 to 2000 $M_{\text {Earth }}, 8$ to $32 R_{\text {Earth }}$ ) and medium (10 to $100 M_{\text {Earth }}, 4$ to $\left.8 R_{\text {Earth }}\right)$ planets.

\section{Discrepancy in $Q_{*}^{\prime}$ from giant and medium radii planets}

When tidal migration equations (e.g., Jackson et al. 2009) operate on an occurrence distribution of planets initially taken to be a single power law, the result produced is a two-power law distribution, with tidal migration producing a short period fall off that follows a steeper power law. In Fig. 1, we compare fits by H12 with fall offs calculated for a range of tidal distributions. H12 used a two-power-law equation to the fit Kepler planet candidates. We show their occurrence distributions for giant and medium radii planets in Fig. 1, plotted against our calculations of occurrence distributions summed for mass after a representive migration time of $4.5 \mathrm{Gyr}$, shown for several values of tidal dissipation strengths $Q_{*}^{\prime}$. The fall off for giant and medium planets could be interpreted as giving different values of $Q_{*}^{\prime}$ for differently sized planets. The difference appears to correspond with the radii where the pile up of giant planets occurs, which suggests that the flow into the pile up raises the occurrence of the shortest period giant planets. This difference appears to be best reconciled by a flow of high eccentricity giant planets creating the pile up (S12), from which an increased number of planets migrate though the shortest period region and into the star.

\section{Future fall in rates consistent only with giants arriving}

In the three panels of Fig. 2 we show, for giant, medium, and relatively small planets, the calculated future in fall rates plotted for stellar tidal dissipation values of $Q_{*}^{\prime}$ from $10^{6.5}$ to $10^{8.5}$. We show here the rate of infall calculated for the fits by H12; infall calculated directly using data directly, though noisy, show the same discrepancy (Taylor 2012 , in preparation). The value of $Q_{*}^{\prime}$ chosen must lead to infall rates that decrease at a rate no faster than the supply of planets can reasonably decrease as the star population
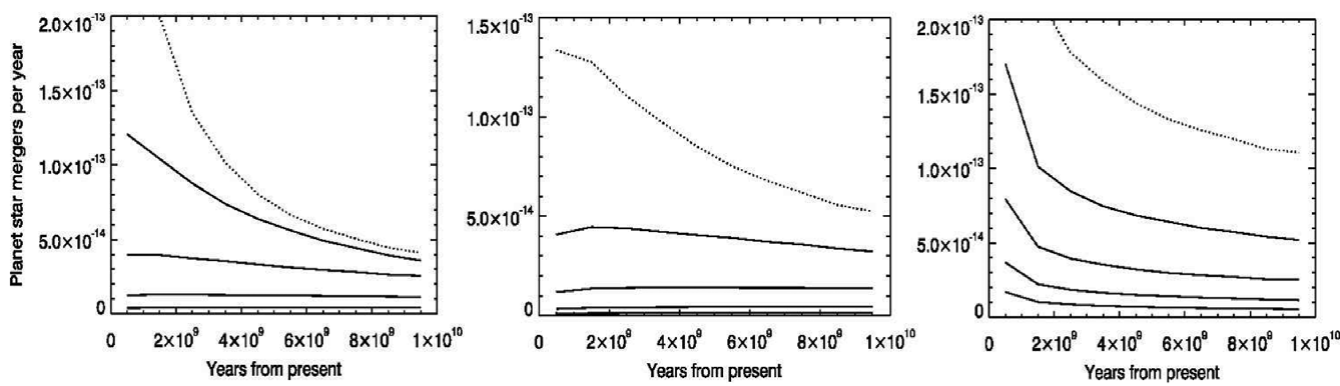

Figure 2. Future fall in rates for the three ranges of planet radii (three panels, for large, medium, small) each with plots for tidal dissipation values of $\log \left(Q_{*}^{\prime}\right)$ values of $10^{6.5}$ (dotted, top), $10^{7.0}$, $10^{7.5}, 10^{8.0}$, and $10^{8.5}$. 


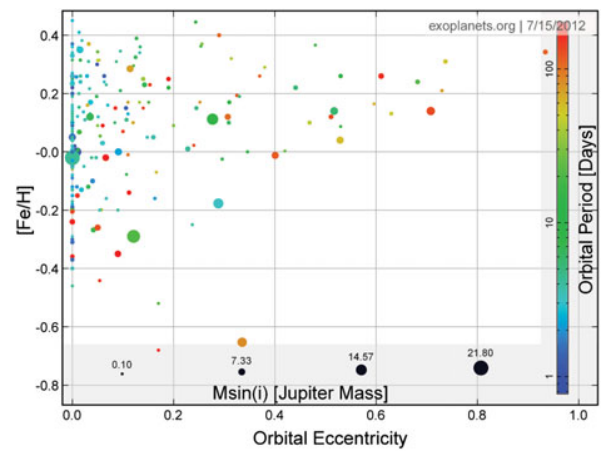

Figure 3. Correlation of increasing $\mathrm{Fe} / \mathrm{H}$ with increasing eccentricity for planets with periods less than 200 days and masses greater than $0.1 M_{J}$. Found using exoplanets.org.

becomes older. Adding an inward flow of giant planets allows an extrapolation of the future rate of infall such that $Q_{*}^{\prime}<10^{7}$ is reasonable, which would be more consistent with the tidal dissipation strength evaluated for medium planets of closer to $Q_{*}^{\prime} \sim 10^{7}$.

The rate of this flow only needs to be on the order of $10^{-12}$ giant planets per year per star for the fall in rates to be consistent, a rate that the population of longer period planets should be able to supply.

\section{Multiplanet pollution, and conclusions}

If inwardly migrating giant planets are more likely to scatter other planets into the star before circularizing, then this might explain why short period giant radii planets are more often the only planet, yet smaller planets are more often in multiple systems (Fabrycky et al. 2012). We present an apparent correlation of higher eccentricity with increasing $[\mathrm{Fe} / \mathrm{H}]$, as shown in Fig. 3. Buchhave et al. (2012) find that stars hosting smaller radii planets do not preferentially have higher $[\mathrm{Fe} / \mathrm{H}]$. Might the migration of large planets have disrupted orbits of smaller planets? The pollution explanation for the correlation of $[\mathrm{Fe} / \mathrm{H}]$ with planets has been opposed based on missing correlations with other elements, but it may be that circularized planets have had more time for the star to have mixed the pollution.

The migration of giant planets into short period orbits may have disrupted the orbits of smaller planets. It is important to understand why the flow of giant planets appears to be higher, but perhaps planets whose orbits have been disrupted migrate more quickly.

We conclude that there appears to be a flow of giant planets into the star that appears to be significantly different from how smaller planets migrate into the star.

\section{References}

Buchhave, L. A., Latham, D. W., Johansen, A., et al. 2012, Nature, 486, 375

Fabrycky, D. C., Lissauer, J. J., et al. 2012, ApJ, submitted (arXiv:astro-ph/1202.6328)

Howard, A. W., Marcy, G. W., Bryson, S. T. et al. 2012, ApJS, 201,15

Jackson, B., Barnes, R., \& Greenberg, R. 2009, ApJ, 698, 1357

Socrates, A., Katz, B., Dong, S., \& Tremaine, S. 2012, ApJ, 750, 106

Taylor, S. F. 2012, European Planetary Science Congress, Abstract id. EPSC2012-784 